Pacific Journal of Mathematics

CLEAR VISIBILITY AND UNIONS OF TWO STARSHAPED 


\title{
CLEAR VISIBILITY AND UNIONS OF TWO STARSHAPED SETS IN THE PLANE
}

\author{
MARILYN BREEN
}

\begin{abstract}
Let $S$ be a compact set in $R^{2}$. Assume that for every finite set $F$ in bdry $S$ there exist points $s$ and $t$ (depending on $F$ ) such that every point of $F$ is clearly visible via $S$ from at least one of $s$ or $t$. Then $S$ is a union of two starshaped sets. If "clearly visible" is replaced by the weaker term "visible", then the result fails.
\end{abstract}

1. Introduction. We begin with some preliminary definitions. Let $S$ be a set in $R^{d}$. For points $x$ and $y$ in $S$, we say $x$ sees $y$ via $S$ ( $x$ is visible from $y$ via $S$ ) if and only if the corresponding segment $[x, y]$ lies in $S$. Point $x$ is clearly visible from $y$ via $S$ if and only if there is some neighborhood $N$ of $x$ such that $y$ sees each point of $S \cap N$ via $S$. Set $S$ is starshaped if and only if there is some point $p$ in $S$ such that $p$ sees each point of $S$ via $S$, and the set of all such points $p$ is called the (convex) kernel of $S$.

A well-known theorem of Krasnosel'skii [5] states that if $S$ is a nonempty compact set in $R^{d}$, then $S$ is starshaped if and only if every $d+1$ points of $S$ are visible via $S$ from a common point. Moreover, points of $S$ may be replaced by boundary points of $S$ to produce a stronger result. Other Krasnosel'skii-type theorems have been obtained for starshaped sets, and in several recent studies ([1], [3], [4]), a helpful tool has been the concept of clearly visible.

Here we use the idea of clearly visible to examine a related problem, that of obtaining a Krasnosel'skii-type characterization for unions of starshaped sets. Although this kind of problem is mentioned in [8, Prob. 6.6, p. 178] and in [2], it is also closely related to work by Lawrence, Hare, and Kenelly [6] concerning unions of convex sets, and their results will play an important role.

Restricting our attention to unions of two starshaped sets in the plane, we establish the following result: Let $S$ be a compact set in $R^{2}$. Assume that for every finite set $F$ in the boundary of $S$ there exist points $S$ and $t$ (depending on $F$ ) such that every point of $F$ is clearly visible via $S$ from at least one of $s$ or $t$. Then $S$ is a union of two starshaped sets. If 
"clearly visible" is replaced by the weaker term "visible", then the result fails. Finally, a general characterization theorem for compact unions of starshaped sets is given.

The following terminology will be used throughout the paper: Conv $S$, cl $S$, int $S$, and bdry $S$ will denote the convex hull, closure, interior, and boundary, respectively, for set $S$. For distinct points $x$ and $y, L(x, y)$ will represent the line through $x$ and $y$, and $\operatorname{dist}(x, y)$ will be the distance from $x$ to $y$. The reader is referred to Valentine [8] and to Lay [7] for a discussion of these concepts.

2. The results. Before establishing the main result, we will present a sequence of four preliminary lemmas adapted from a theorem by Lawrence, Hare, and Kenelly [6, Theorem 1]. For simplicity of notation, these results are stated for pairs of sets. However, each has an immediate analogue for $k$-tuples of sets as well.

Definition 1. Let $T$ be a collection whose members are unordered pairs of sets. We say that a collection $M$ of ordered pairs is a pairing for $T$ if and only if the following hold:

(1) For every $(C, D)$ in $M,\{C, D\}$ is in $T$.

(2) For every $\{C, D\}$ in $T$, exactly one of the ordered pairs $(C, D)$, $(D, C)$ is in $M$.

LEMMA 1. Let $\mathscr{P}$ be a property meaningful for finite collections of ordered pairs of sets, and let $T$ be a collection of unordered pairs of sets. If every finite subset of $T$ has a pairing satisfying property $\mathscr{P}$, then $T$ has a pairing $M$ such that every finite subset of $M$ satisfies property $\mathscr{P}$.

Proof. The argument is adapted from [6, Theorem 1] and is included for completeness. Let $\mathscr{F}$ be the family of all finite subsets of $T$. Then for every $F$ in $\mathscr{F}$, there corresponds a suitable pairing. (That is, there corresponds a pairing for $F$ having property $\mathscr{P}$.) We let $P_{F}$ denote the collection of all suitable pairings for $F$. Observe that since $F$ is finite, so is $P_{F}$, and $P_{F}$ with the discrete topology is compact. By the Tychonoff theorem, the product $\pi P_{F}$ is compact, too. For $X$ in the product, let $X_{F}$ denote its $F$ th coordinate, and for $G$ in $\mathscr{F}$, define $A_{G} \equiv\left\{X\right.$ in $\pi P_{F}$ : if $H \subseteq G$, then $\left.X_{H}=\left.X_{G}\right|_{H}\right\}$, where $\left.X_{G}\right|_{H}$ means $X_{G}$ restricted to $H$.

We assert that $\left\{A_{G}: G\right.$ in $\left.\mathscr{F}\right\}$ is a collection of compact sets having the finite intersection property: It is not hard to show that each $A_{G}$ is closed (hence compact) and nonempty. To see that $\left\{A_{G}: G\right.$ in $\left.\mathscr{F}\right\}$ has the 
finite intersection property, observe that when $F_{1}, \ldots, F_{n} \in \mathscr{F}$, then $A_{F_{1}} \cap$ $\cdots \cap A_{F_{n}}$ contains $A_{F_{1} \cup \cdots \cup F_{n}} \neq \varnothing$. Hence $\bigcap\left\{A_{G}: G\right.$ in $\left.\mathscr{F}\right\} \neq \varnothing$, and we may select $Z$ in this intersection. Notice that for every $H$ and $G$ in $\mathscr{F}$ with $H \subseteq G, Z_{H}=\left.Z_{G}\right|_{H}$.

Finally, for every pair $\{C, D\}$ in $T$, let $F(C, D)$ denote the member of $\mathscr{F}$ consisting of $\{C, D\}$ only. Then $Z_{F(C, D)}$ is a suitable pairing, say $(C, D)$, for $F(C, D)$, and whenever $F(C, D) \subseteq G$ for $G$ in $\mathscr{F}$, then $Z_{F(C, D)}$ and $Z_{G}$ agree on $F(C, D)$. Letting $M$ be the set of ordered pairs $(C, D)$ such that $\{(C, D)\}=Z_{F(C, D)}$ for some pair $\{C, D\}$ in $T$, a standard argument shows that $M$ satisfies the lemma.

LEMMA 2. Let $S$ be a compact set in $R^{d}, Q$ a finite subset of $S$, and let $M \equiv\left\{\left(C_{l}, D_{i}\right): 1 \leq i\right\}$ be a family of ordered pairs of closed sets. Assume that for every $j$ there exists a partition $\left\{Q_{j 1}, Q_{j 2}\right\}$ of $Q$ such that each point of $Q_{j 1}$ sees via $S$ a common point of $\cap\left\{C_{i}: 1 \leq i \leq j\right\}$ and each point of $Q_{j 2}$ sees via $S$ a common point of $\cap\left\{D_{i}: 1 \leq i \leq j\right\}$. Then there is a partition $\left\{Q_{1}^{\prime}, Q_{2}^{\prime}\right\}$ of $Q$ such that each point of $Q_{1}^{\prime}$ sees via $S$ a common point of $\cap\left\{C_{i}: 1 \leq i\right\}$ and each point of $Q_{2}^{\prime}$ sees via $S$ a common point of $\bigcap\left\{D_{i}: 1 \leq i\right\}$.

Proof. Again the argument is adapted from [6, Theorem 1]. For every $j$, let $P_{j}$ denote the set of all ordered pairs $\left(Q_{j 1}, Q_{j 2}\right)$, where $\left\{Q_{j 1}, Q_{j 2}\right\}$ is a partition of $Q, Q_{j 1}$ sees a common point of $\bigcap\left\{C_{i}: 1 \leq i \leq j\right\}$, and $Q_{j 2}$ sees a common point of $\bigcap\left\{D_{i}: 1 \leq i \leq j\right\}$. Using the fact that $Q$ is finite, we see that $P_{j}$ is finite, $P_{J}$ is compact with the discrete topology, and the product $\pi P_{j}$ is compact. Let $X_{j}$ denote the $j$ th coordinate of $X$ in $\pi P_{j}$, and for each $k$, define set $A_{k} \equiv\left\{X\right.$ in $\pi P_{j}: X_{i}=X_{k}$ for $\left.i \leq k\right\}$. Using an argument like the one in Lemma $1,\left\{A_{k}: 1 \leq k\right\}$ is a family of compact sets having the finite intersection property, so we may select some $Z$ in $\bigcap\left\{A_{k}: 1 \leq k\right\}$. Then for every $i$ and $j, Z_{l}=Z_{j}$, and we let $\left(Q_{1}^{\prime}, Q_{2}^{\prime}\right)$ denote this common value.

We assert that $\left\{Q_{1}^{\prime}, Q_{2}^{\prime}\right\}$ satisfies the lemma: For each $j$, select a point $c_{j}$ in $\cap\left\{C_{i}: 1 \leq i \leq j\right\}$ such that $Q_{1}^{\prime}$ sees $c_{j}$ via $S$. Since $S$ is compact, the sequence $\left\{c_{j}: 1 \leq j\right\}$ has a limit point $c$ in $S$. Moreover, it is easy to verify that $c \in \bigcap\left\{C_{j}: 1 \leq j\right\}$ and that each point of $Q_{1}^{\prime}$ sees $c$ via $S$. Parallel statements hold for $Q_{2}^{\prime}$ and some $d \in \bigcap\left\{D_{j}: 1 \leq j\right\}$, and the lemma is established.

The next lemma is a slightly stronger version of [6, Theorem 1]. The proofs are essentially the same. 
Lemma 3 (Lawrence, Hare, Kenelly Lemma). For $i=1,2$, let $P_{i}$ be a hereditary property of sets. Let $B$ be a set such that for every finite subset $F \subseteq B$, there is a partition $\left\{F_{1}, F_{2}\right\}$ of $F$ such that $F_{i}$ has property $P_{i}$, $i=1,2$. Then there is a partition $\left\{B_{1}, B_{2}\right\}$ of $B$ such that every finite subset of $B_{i}$ has property $P_{i}, i=1,2$.

LEMMA 4. Let $S$ be a compact set in some linear topological space. Suppose that every finite set $F$ in bdry $S$ may be partitioned into two sets $F_{1}$ and $F_{2}$ such that each point of $F_{i}$ is visible via $S$ from a point in the closed set $C_{i}, i=1,2$. Then bdry $S$ may be partitioned into two sets $S_{1}$ and $S_{2}$ such that each point in $S_{i}$ is visible via $S$ from a point in $C_{l}, i=1,2$.

Proof. By the Lawrence, Hare, Kenelly Lemma, there is a partition $\left\{S_{1}, S_{2}\right\}$ of bdry $S$ such that every finite subset of $S_{i}$ is visible via $S$ from a common point of $C_{i}, i=1,2$. For every finite subset $G$ of $S_{1}$, let $A_{G}$ denote the subset of $C_{1}$ seeing $G$ via $S$. Standard arguments yield a point $c_{1} \in \bigcap\left\{A_{G}: G\right.$ finite, $\left.G \subseteq S_{1}\right\} \neq \varnothing$, and each point of $S_{1}$ is visible via $S$ from $c_{1}$. A parallel argument holds for $S_{2}$, and the lemma is proved.

We are ready to state our main theorem.

THEOREM 1. Let $S$ be a compact set in $R^{2}$. Assume that for every finite set $F$ in bdry $S$ there exist points $s$ and $t$ (depending on $F$ ) such that every point of $F$ is clearly visible via $S$ from at least one of $s$ or $t$. Then $S$ is a union of two starshaped sets.

Proof. The proof will require an intermediate result concerning bounded components of $R^{2}-S$.

LEMMA 5. If $J$ and $K$ are bounded components of $R^{2}-S$ with conv $J \cap$ $\operatorname{conv} K=\varnothing$, then $\operatorname{clconv} J \cap \operatorname{clconv} K=\varnothing$.

Proof of Lemma 5. Suppose on the contrary that cl conv $J \cap \operatorname{clconv} K$ $\neq \varnothing$. Since these sets share no interior points, they may be separated by a line $L$, and clearly

$$
\text { bdry conv } J \cap \text { bdry conv } K=\operatorname{cl} \operatorname{conv} J \cap \operatorname{cl} \operatorname{conv} K \subseteq L .
$$

Moreover, it is not hard to show that for an appropriate labeling of $J$ and $K$, bdry $J \cap$ bdry conv $K \neq \varnothing$. Let $x$ be a point in this nonempty intersection. Clearly $x \in$ bdry $S \cap L$. Let $L^{\prime}$ and $L^{\prime \prime}$ be lines distinct from $L$ and parallel to $L$, with $L^{\prime}$ supporting clconv $J$ and $L^{\prime \prime}$ supporting clconv $K$. 
By standard arguments, $L^{\prime}$ meets bdry conv $J$ at some point $y$ in bdry $J \subseteq$ bdry $S$, and similarly $L^{\prime \prime}$ meets bdry conv $K$ at some $z$ in bdry $K \subseteq$ bdry $S$. By our hypothesis in Theorem 1, two points from $\{x, y, z\}$ must be clearly visible from a common point of $S$. However, it is easy to show that this cannot occur. Our supposition is false, the sets are disjoint, and the lemma is established.

We are ready to prove Theorem 1 , and we begin by defining special points $c$ and $d$ in $S$ which will satisfy the theorem. Assume for the moment that $R^{2}-S$ has at least two bounded components $A$ and $B$ with $\operatorname{conv} A \cap$ $\operatorname{conv} B=\varnothing$. By Lemma $5, \operatorname{cl} \operatorname{conv} A \cap \operatorname{cl} \operatorname{conv} B=\varnothing$. Hence there are distinct lines $L(A, B) \equiv L$ and $N(A, B) \equiv N$ such that each line supports both cl conv $A$ and $\operatorname{clconv} B$, with $A$ and $B$ in opposite open halfplanes. Standard arguments may be used to produce points $a_{1} \in L \cap$ bdry $A \subseteq$ bdry $S$ and $b_{1} \in L \cap$ bdry $B \subseteq$ bdry $S$ with $\operatorname{dist}\left(a_{1}, b_{1}\right)$ maximal. Similarly, choose $a_{2} \in N \cap$ bdry $A$ and $b_{2} \in N \cap$ bdry $B$ with $\operatorname{dist}\left(a_{2}, b_{2}\right)$ maximal. Label the open halfplanes determined by $L$ and $N$ so that $b_{2} \in L_{2}$ and $a_{1} \in N_{1}$. It is easy to see that $b_{1}$ is clearly visible only from point in $\mathrm{cl} L_{1}, a_{1}$ only from points in $\mathrm{cl} L_{2}, b_{2}$ only from points in $\mathrm{cl} N_{1}$ and $a_{2}$ only from points in $\mathrm{cl} N_{2}$.

A simple geometric argument may be used to find point $a_{3}$ in bdry $A \subseteq$ bdry $S$ not clearly visible from any point of $\mathrm{cl} N_{2} \cap \mathrm{cl} L_{2}$ and point $b_{3}$ in bdry $B \subseteq$ bdry $S$ not clearly visible from any point of cl $N_{1} \cap$ cl $L_{1}$ : Precisely, let line $H$ bisect the angles determined by $\operatorname{cl} L_{1} \cap \mathrm{cl} N_{2}$ and $\mathrm{cl} L_{2} \cap \mathrm{cl} N_{1}$. Let $H^{\prime}$ and $H^{\prime \prime}$ be lines parallel to $H$ such that $H^{\prime}$ supports $\operatorname{conv} A$ at some $a_{3} \in \operatorname{bdry} A$ and $H^{\prime \prime}$ supports $\operatorname{conv} B$ at some $b_{3} \in$ bdry $B$. Then $a_{3}$ is not clearly visible via $S$ from any point of cl $N_{2} \cap \mathrm{cl} L_{2}, b_{3}$ is not clearly visible via $S$ from any point of $\mathrm{cl} N_{1} \cap \mathrm{cl} L_{1}$, and $a_{3}$ and $b_{3}$ are not clearly visible from any common point of $S$.

Finally, let $Q(A, B) \equiv\left\{a_{i}, b_{i}:: 1 \leq i \leq 3\right\}$. By hypothesis, there exist points $s$ and $t$ of $S$ such that each point of $Q(A, B)$ is clearly visible via $S$ from one of $s$ or $t$. By comments above, $s$ and $t$ must lie in opposite closed halfplanes determined by each of $L$ and $N$, neither is in $\mathrm{cl} N_{1} \cap \mathrm{cl} L_{1}$ or cl $N_{2} \cap \mathrm{cl} L_{2}$, so for an appropriate labeling of $s$ and $t, s \in \mathrm{cl} N_{1} \cap \mathrm{cl} L_{2}$ and $t \in \operatorname{cl} N_{2} \cap \mathrm{cl} L_{1}$. Define $C(A, B) \equiv \operatorname{cl~} N_{1} \cap \mathrm{cl} L_{2}$ and $D(A, B) \equiv$ cl $N_{2} \cap \mathrm{cl} L_{1}$. In the future we shall refer to $C(A, B)$ and $D(A, B)$ as opposite vertical angles associated with $A$ and $B$.

For every pair of distinct components $A$ and $B$ in $R^{2}-S$ satisfying $\operatorname{conv} A \cap \operatorname{conv} B=\varnothing$, define sets $Q(A, B), C(A, B), D(A, B)$ in the manner described above, and let $T$ be the set consisting of all unordered pairs $\{C(A, B), D(A, B)\}$. Let $Q$ be a fixed subset of bdry $S, Q$ finite. 
Observe that if $\left\{\left\{C\left(A_{i}, B_{i}\right), D\left(A_{i}, B_{i}\right)\right\}: 1 \leq i \leq n\right\}$ is any finite subset of $T$, then $Q^{\prime} \equiv Q \cup Q\left(A_{1}, B_{1}\right) \cup \cdots \cup Q\left(A_{n}, B_{n}\right)$ is finite. Hence by hypothesis there exist points $s^{\prime}$ and $t^{\prime}$ such that every point of $Q^{\prime}$ is clearly visible via $S$ from one of $s^{\prime}$ or $t^{\prime}$. Moreover, by comments above, for an appropriate labeling of the corresponding sets $C\left(A_{i}, B_{i}\right)$ and $D\left(A_{i}, B_{i}\right)$, $s^{\prime} \in \bigcap\left\{C\left(A_{i}, B_{i}\right): 1 \leq i \leq n\right\}$ and $t^{\prime} \in \bigcap\left\{D\left(A_{i}, B_{l}\right): 1 \leq i \leq n\right\}$.

We define property $\mathscr{P}$ as follows: For $T^{\prime}$ a finite subset of $T$ and $M^{\prime}=\left\{\left(C_{1}, D_{1}\right), \ldots,\left(C_{n}, D_{n}\right)\right\}$ a pairing for $T^{\prime}$, we say that $M^{\prime}$ has property $\mathscr{P}$ if and only if there exists a partition $\left\{Q_{1}, Q_{2}\right\}$ of $Q$ such that each point of $Q_{1}$ sees via $S$ a common point of $\bigcap\left\{C_{i}: 1 \leq i \leq n\right\}$ and each point of $Q_{2}$ sees via $S$ a common point of $\cap\left\{D_{i}: 1 \leq i \leq n\right\}$. By comments above, every finite subset of $T$ has a pairing satisfying property $\mathscr{P}$. Therefore, we may use Lemma 1 to conclude that $T$ has a pairing $M$ such that every finite subset of $M$ satisfies property $\mathscr{P}$. Since $R^{2}-S$ has at most countably many bounded components, $M$ is countable, and we let $M=\left\{\left(C_{i}, D_{i}\right): 1 \leq i\right\}$. Furthermore, sets $S, Q$, and $M$ satisfy the hypothesis of Lemma 2, so there exists a partition $\left\{Q_{1}^{\prime}, Q_{2}^{\prime}\right\}$ of $Q$ such that each point of $Q_{1}^{\prime}$ sees via $S$ a common point of $\cap\left\{C_{i}: 1 \leq i\right\}$ and each point of $Q_{2}^{\prime}$ sees via $S$ a common point of $\bigcap\left\{D_{i}: 1 \leq i\right\}$. Hence we may apply Lemma 4 to conclude that there is a partition $\left\{S_{1}, S_{2}\right\}$ for bdry $S$ such that each point of $S_{1}$ is visible via $S$ from a common point $c$ of $\cap\left\{C_{i}: 1 \leq i\right\}$ and each point of $S_{2}$ is visible via $S$ from a common point $d$ of $\cap\left\{D_{i}: 1 \leq i\right\}$.

We have defined points $c$ and $d$ in case $R^{2}-S$ contains two bounded components $A$ and $B$ with $\operatorname{conv} A \cap \operatorname{conv} B=\varnothing$. In case no such components exist, then by Lemma 4 simply choose points $c$ and $d$ in $S$ such that each point of bdry $S$ sees via $S$ either $c$ or $d$.

To complete the proof, we will show that every point of $S$ sees via $S$ either $c$ or $d$. Let $x \in S$ and suppose that neither $c$ nor $d$ sees $x$, to reach a contradiction. Clearly $x$ must be an interior point of $S$. Choose the segment at $x$ in $S \cap L(c, x)$ having maximal length, and let $p$ and $q$ denote its endpoints, with $c<p<x<q$. Then $p, q \in$ bdry $S, c$ sees neither $p$ nor $q$ via $S$, so $d$ must see both $p$ and $q$ via $S$. Observe that $d \notin L(c, x)$ since $d$ cannot see $x$. Similarly, choose a segment at $x$ in $S \cap L(d, x)$ having maximal length, and let $r$ and $s$ denote its endpoints, $d<r<x<s$. Then $c$ sees via $S$ both $r$ and $s$. (See Figure 1)

Since $d$ does not see $x$ via $S$, there is a segment in $(d, r)-S$, and this segment belongs to a bounded component $K$ of $R^{2}-S, K \subseteq$ int $\operatorname{conv}\{p, q, d\}$. Likewise, there is a segment in $(c, p)-S$ belonging to 


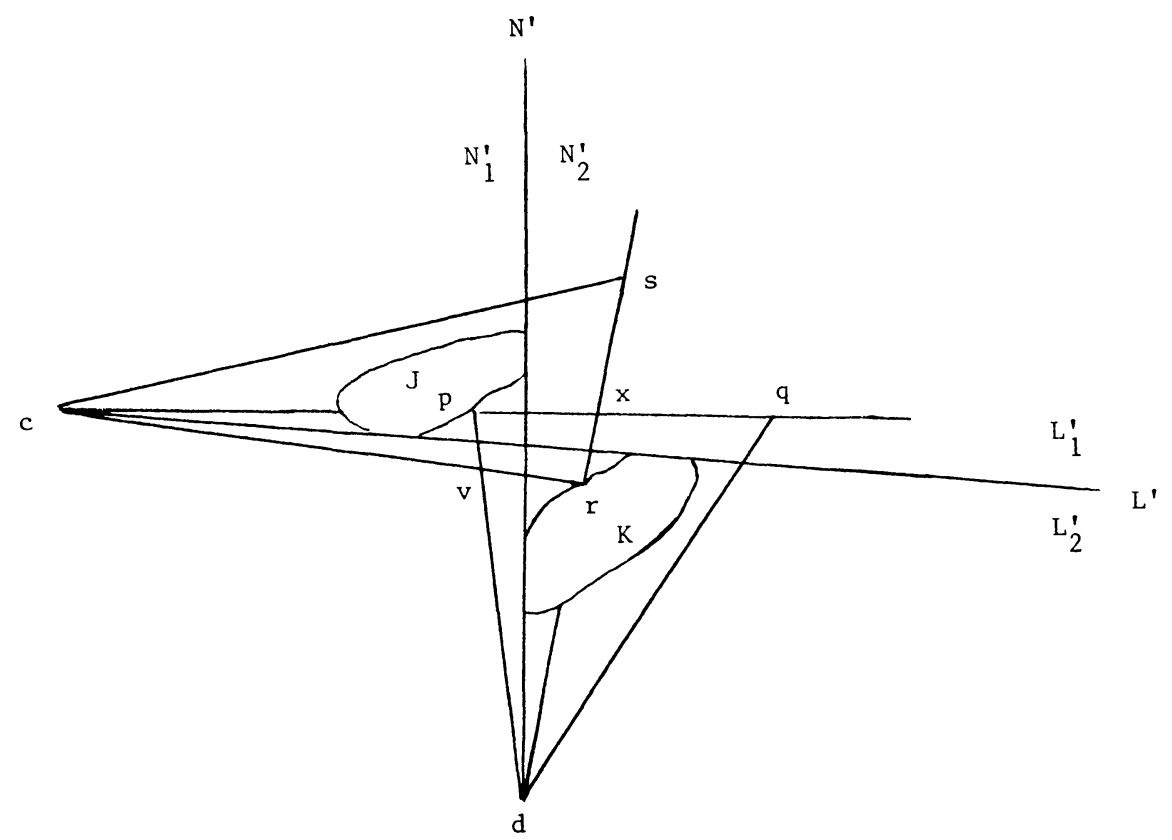

FiguRE 1

a bounded component $J$ of $R^{2}-S, J \subseteq$ int $\operatorname{conv}\{c, s, r\}$. Letting $L(c, r)$ $\cap L(d, p)=\{v\}$, it is easy to show that $J$ and $K$ lie in opposite open halfplanes determined by $L(v, x)$, so conv $J \cap$ conv $K=\varnothing$. Hence points $c$ and $d$ must have been selected according to the lengthy procedure described in previous paragraphs.

Define lines $L^{\prime}$ and $N^{\prime}$ as follows: Clearly $L(c, v) \cap J=\varnothing$. If $L(c, v) \cap K=\varnothing$ as well, let $L^{\prime}=L(c, v)$ and let $L_{1}^{\prime}$ be the open halfplane determined by $L^{\prime}$ and containing $J$. Then $K \subseteq L_{2}^{\prime}$. Otherwise, $L(c, v)$ meets $K$. In this case, let $L_{1}$ denote the open halfplane determined by $L(c, v)$ and containing $J$. Clearly $d$ cannot see all the points of (bdry $K) \cap L_{1}$, so $c$ must see some of these points via $S$. Let $L^{\prime}$ be the line from $c$ which supports conv $K$ at a point of $L_{1}$. It is easy to show that $L^{\prime} \cap($ bdry conv $K)$ contains some point $t$ of bdry $K$ such that $[c, t] \subseteq S$. Thus $[c, t] \cap J=\varnothing$, and $J$ lies in the open halfplane $L_{1}^{\prime}$ determined by $L^{\prime}$ and containing $p$. Of course $K$ lies in the opposite halfplane $L_{2}^{\prime}$.

Using a similar argument, $L(d, v) \cap K=\varnothing$. If $L(d, v) \cap J=\varnothing$ as well, let $N^{\prime}=L(d, v)$ and let $N_{2}^{\prime}$ denote the open halfplane determined by $N^{\prime}$ and containing $K$. Then $J \subseteq N_{1}^{\prime}$. Otherwise, $L(d, v)$ meets $J$. In this case, let $N_{2}$ denote the open halfplane determined by $L(d, v)$ and containing $K$. Clearly $d$ must see via $S$ some points of (bdry $J) \cap N_{2}$. Let $N^{\prime}$ 
be the line from $d$ which supports conv $J$ at a point of $N_{2}$. Then $N^{\prime} \cap\left(\right.$ bdry conv $J$ ) contains some point $t^{\prime}$ of bdry $J$ such that $\left[d, t^{\prime}\right] \subseteq S$. Thus $\left[d, t^{\prime}\right] \cap K=\varnothing$ and $K$ lies in the open halfplane $N_{2}^{\prime}$ determined by $N$ and containing $r$. Of course $J$ lies in the opposite halfplane $N_{1}^{\prime}$.

We have $J \subseteq N_{1}^{\prime} \cap L_{1}^{\prime}$ and $K \subseteq N_{2}^{\prime} \cap L_{2}^{\prime}$. Observe that if line $H$ meets both cl $J$ and $\mathrm{cl} K$, then $H \cap \mathrm{cl} N_{1}^{\prime} \cap \mathrm{cl} L_{1}^{\prime}$ is an infinite ray, as is $H \cap$ cl $N_{2}^{\prime} \cap \operatorname{cl} L_{2}^{\prime}$. Moreover, $c$ and $d$ must lie in the same closed halfplane determined by $H$.

Recall that since conv $J \cap \operatorname{conv} K=\varnothing$, we have associated with $J$ and $K$ distinct lines $L(J, K)$ and $N(J, K)$ which support both cl conv $J$ and cl conv $K$, with $J$ and $K$ in opposite closed halfplanes determined by each line. Further, by our choice of $c \in \bigcap\left\{C_{i}: 1 \leq i\right\}$ and $d \in$ $\cap\left\{D_{i}: 1 \leq i\right\}, c$ and $d$ belong to opposite vertical angles $C(J, K)$ and $D(J, K)$ associated with $J$ and $K$. However, our comments in the preceding paragraph (concerning line $H$ ) imply that $c$ and $d$ must lie in the same vertical angle, either $C(J, K)$ or $D(J, K)$. The only way for both these events to occur is for $c$ and $d$ to be the same point, impossible since $d \notin L(c, x)$. Our supposition (that neither $c$ nor $d$ sees $x$ ) must be false, and $S$ is indeed a union of two starshaped sets. This finishes the proof of the theorem.

It is easy to find examples to show that the condition in Theorem 1 does not characterize unions of starshaped sets: Consider a $W$-shaped polygonal path.

Furthermore, it is interesting to observe that if the words "clearly visible" in Theorem 1 are replaced by the weaker term "visible", then the result fails, as the following example illustrates.

EXAMPLE 1. Let $S$ be the compact set in Figure 2, with shaded regions in $R^{2}-S$ and dotted segments in $S$. Then every boundary point of $S$ is visible via $S$ from either $c$ or $d$, yet $S$ is not a union of two starshaped sets.

However, if in Theorem 1 we replace "clearly visible" by "visible" and require $S$ to be simply connected, then the result holds. The easy proof is a simplified version of our previous argument.

We close with a theorem concerning unions of starshaped sets which follows easily from work by Lawrence, Hare, and Kenelly.

THEOREM 2. Let $S$ be a compact set in some linear topological space. Then $S$ is a union of $k$ starshaped sets if and only if for every finite set $F$ in $S$ there exist points $s_{1}, \ldots, s_{k}$ (depending on $F$ ) such that each point of $F$ sees via $S$ at least one of the $s_{i}$ points. 


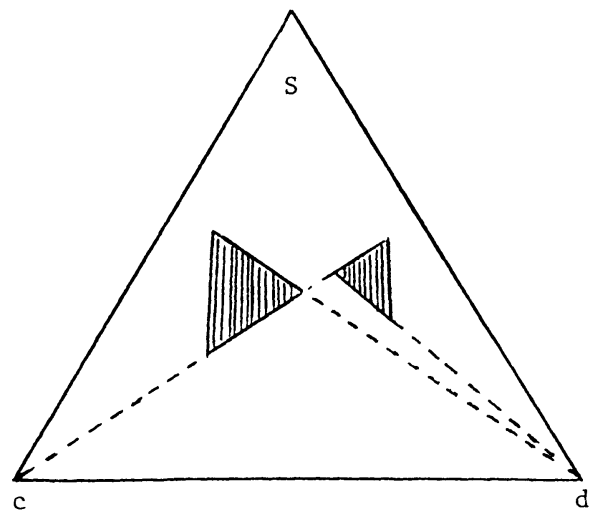

FIGURE 2

Proof. The necessity is immediate. For the sufficiency, apply [6, Theorem 1] to obtain a $k$-partition $\left\{S_{1}, \ldots, S_{k}\right\}$ of $S$ such that each finite subset of $S_{l}$ is visible from a common point of $S, 1 \leq i \leq k$. By standard arguments, every point of $S_{i}$ is visible from a common point of $S$, and the theorem is established.

\section{REFERENCES}

[1] Marilyn Breen, A Krasnosel'skii-type theorem for points of local nonconvexity, Proc. Amer. Math. Soc., 85 (1982), 216-266.

[2] An example concerning unions of two starshaped sets in the plane, Israel J. Math., 17 (1974), 347-349.

[3] Clear visibility and the dimension of kernels of starshaped sets; Proc. Amer. Math. Soc., 85 (1982), 414-418.

[4] _ Clear visibility, starshaped sets, and finitely starlike sets, J. Geometry, 19 (1982), 183-196.

[5] M. A. Krasnosel'skii, Sur un critère pour qu'un domain soit étoilé, Math. Sb., 19 (61) (1946), 309-310.

[6] J. F. Lawrence, W. R. Hare, Jr. and John W. Kenelly, Finite unions of convex sets, Proc. Amer. Math. Soc., 34 (1972), 225-228.

[7] Steven R. Lay, Convex Sets and Their Applications, John Wiley, New York, 1982.

[8] F. A. Valentine, Convex Sets, McGraw Hill, New York, 1964.

Received March 14, 1983

UNIVERSITY OF OKLAHOMA

NORMAN, OK 73019 



\title{
PACIFIC JOURNAL OF MATHEMATICS EDITORS
}

\author{
Donald BABBITt (Managing Editor) \\ University of California \\ Los Angeles, CA 90024 \\ J. DugunduI \\ University of Southern California \\ Los Angeles, CA 90089-1113 \\ R. FINN \\ Stanford University \\ Stanford, CA 94305 \\ HermanN FlaschKa \\ University of Arizona \\ Tucson, AŻ 85721
}

C. C. Moore

University of California

Berkeley, CA 94720

ARTHUR Ogus

University of California

Berkeley, CA 94720

Hugo Rossi

University of Utah

Salt Lake City, UT 84112

H. SAMELSON

Stanford University

Stanford, CA 94305

ASSOCIATE EDITORS
R. ARENS
E. F. BECKENBACH
B. H. NEUMANN
F. WOLF
K. YoSHIDA (1906-1982)

\section{SUPPORTING INSTITUTIONS}

$\begin{array}{ll}\text { UNIVERSITY OF ARIZONA } & \text { UNIVERSITY OF OREGON } \\ \text { UNIVERSITY OF BRITISH COLUMBIA } & \text { UNIVERSITY OF SOUTHERN CALIFORNIA } \\ \text { CALIFORNIA INSTITUTE OF TECHNOLOGY } & \text { STANFORD UNIVERSITY } \\ \text { UNIVERSITY OF CALIFORNIA } & \text { UNIVERSITY OF HAWAII } \\ \text { MONTANA STATE UNIVERSITY } & \text { UNIVERSITY OF TOKYO } \\ \text { UNIVERSITY OF NEVADA, RENO } & \text { UNIVERSITY OF UTAH } \\ \text { NEW MEXICO STATE UNIVERSITY } & \text { WASHINGTON STATE UNIVERSITY } \\ \text { OREGON STATE UNIVERSITY } & \text { UNIVERSITY OF WASHINGTON }\end{array}$

The Supporting Institutions listed above contribute to the cost of publication of this Journal, but they are not owners or publishers and have no responsibility for its content or policies.

Mathematical papers intended for publication in the Pacific Journal of Mathematics should be in typed form or offset-reproduced (not dittoed), double spaced with large margins. Please do not use built up fractions in the text of the manuscript. However, you may use them in the displayed equations. Underline Greek letters in red, German in green, and script in blue. The first paragraph must be capable of being used separately as a synopsis of the entire paper. In particular it should contain no bibliographic references. Please propose a heading for the odd numbered pages of less than 35 characters. Manuscripts, in triplicate, may be sent to any one of the editors. Please classify according to the scheme of Math. Reviews, Index to Vol. 39. Supply name and address of author to whom proofs should be sent. All other communications should be addressed to the managing editor, or Elaine Barth, University of California, Los Angeles, California 90024.

There are page-charges associated with articles appearing in the Pacific Journal of Mathematics. These charges are expected to be paid by the author's University, Government Agency or Company. If the author or authors do not have access to such Institutional support these charges are waived. Single authors will receive 50 free reprints; joint authors will receive a total of 100 free reprints. Additional copies may be obtained at cost in multiples of 50 .

The Pacific Journal of Mathematics is issued monthly as of January 1966. Regular subscription rate: $\$ 190.00$ a year (5 Vols., 10 issues). Special rate: $\$ 66.00$ a year to individual members of supporting institutions.

Subscriptions, orders for numbers issued in the last three calendar years, and changes of address should be sent to Pacific Journal of Mathematics, P.O. Box 969, Carmel Valley, CA 93924, U.S.A. Old back numbers obtainable from Kraus Periodicals Co., Route 100, Millwood, NY 10546.

The Pacific Journal of Mathematics at P.O. Box 969, Carmel Valley, CA 93924 (ISSN 0030-8730) publishes 5 volumes per year. Application to mail at Second-class postage rates is pending at Carmel Valley, California, and additional mailing offices. Postmaster: Send address changes to Pacific Journal of Mathematics, P.O. Box 969, Carmel Valley, CA 93924.

PUBLISHED BY PACIFIC JOURNAL OF MATHEMATICS, A NON-PROFIT CORPORATION

Copyright $@ 1984$ by Pacific Journal of Mathematics 


\section{Pacific Journal of Mathematics}

\section{Vol. 115, No. $2 \quad$ October, 1984}

Ersan Akyildiz, Gysin homomorphism and Schubert calculus ...........257

Marilyn Breen, Clear visibility and unions of two starshaped sets in the

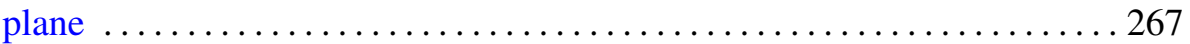

Robert F. Brown, Retraction methods in Nielsen fixed point theory . ......277

Herbert Busemann and Bhalchandra B. Phadke, A general version of Beltrami's theorem in the large ............................... 299

Gerald Arthur Edgar and Robert Francis Wheeler, Topological properties of Banach spaces ............................... 317

Yaakov Friedman and Bernard Russo, Conditional expectation without

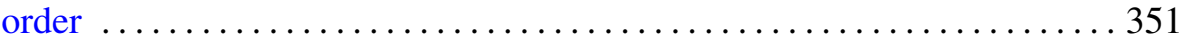

Robert Allen Goggins, Cobordism of manifolds with strong almost tangent

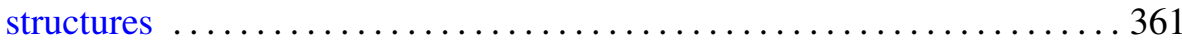

Mike Hoffman, Noncoincidence index of manifolds . . . . . . . . . . . . . 373

William H. Julian, $\varepsilon$-continuity and monotone operations $\ldots \ldots \ldots \ldots 385$

Gerasimos E. Ladas, Y. G. Sficas and I. P. Stavroulakis, Nonoscillatory

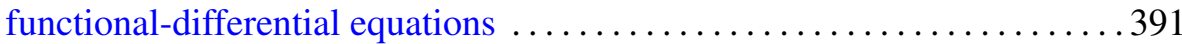

Arnold William Miller and Karel Libor Prikry, When the continuum has

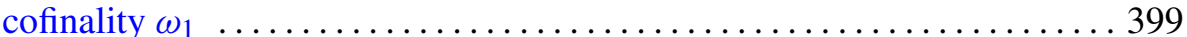

Jean-Leah Mohrherr, Density of a final segment of the truth-table

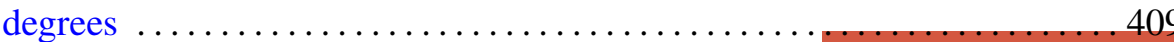

Carl Norman Mutchler, The flat Cauchy problem for radially hyperbolic operators from a characteristic manifold of high codimension ...

Kenji Nakagawa, On the orders of automorphisms of a closed Riemann surface

W. Ricker, Representation of vector-valued functions by Laplace transforms

Jorge Donato Samur, On semigroups of convolution operators in Hilbert

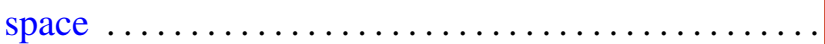

Joseph Gail Stampfli, One-dimensional perturbations of operators 481 Andrew George Earnest and John Sollion Hsia, Correction to: "Spinor norms of local integral rotations. II" 